\title{
Synergistic interaction of phenylpropanoids with antibiotics against bacteria
}

\author{
Shanmugam Hemaiswarya and Mukesh Doble \\ Department of Biotechnology, Indian Institute of Technology Madras, Chennai 600 036, India
}

Correspondence

Mukesh Doble

mukeshd@iitm.ac.in

Received 20 May 2010

Accepted 16 August 2010

\section{INTRODUCTION}

Drug-resistant micro-organisms are a growing global problem as they cause severe infections which may lead to morbidity and mortality. There are a number of ways to reduce the development of drug resistance, and the most important amongst them is to decrease the dose of antibiotics used in the treatment. This can be achieved by using combinations of antibiotics. So far, many such combinations have been studied and applied clinically (Betrosian \& Douzinas, 2009) but the interaction of antibiotics with botanical medicines is less understood. Drug-herb interactions are a major concern for many health-care practitioners. There is also a chance of the interaction of food and its metabolites with synthetic drugs, and such interplay can either be positive or negative.

Phenylpropanoids belong to one such major family of 'polyphenols' which is consumed in our daily diet. They are classified into simple and complex forms, where cinnamic acids and their derivatives fall under the former class. Hydroxycinnamic acids, including caffeic, ferulic, sinapic and $p$-coumaric acids, are present in a large variety of fruits and vegetables, including blueberries, grapes,

Abbreviations: ABTKC, area under the curve for control and treated; AUTKC, area under the time-kill curve; FIC, fractional inhibitory concentration; QSAR, quantitative structure-activity relationship.

A table showing the MICs of antibiotics and phenylpropanoids against various bacteria is available with the online version of this paper. apples, cereal brans, broccoli, spinach and lettuce (Clifford, 1999). The most abundant hydroxycinnamic acid in food is chlorogenic acid, which is an ester of caffeic and quinic acids. These phenylpropanoids interact with many cellular processes and directly influence human health (Korkina, 2007). They can function as antioxidants due to the presence of multiple hydroxyl groups and unsaturated double bonds that can react with radicals and oxidative ions in the cells. The benzene or phenol ring structure in the phenylpropanoids helps them to cross cellular membranes and exert their biological activities. The antibacterial activity of cinnamic acid derivatives has been well studied with main emphasis on the phenyl ring or carboxyl group present in them (Tonari et al., 2002). Owing to the importance of the cinnamic acids as antibacterial agents, the present study was designed to understand the interaction of a few of these with different classes of antibiotics against Gram-positive and -negative bacteria using chequerboard and time-kill curve assays. An attempt is also made to understand the mechanism of action of these phytochemicals and the structural features required for their activity.

\section{METHODS}

Bacterial strains. The strains used in this study were Escherichia coli NCIM 2931, Enterobacter aerogenes NCIM 5139, Pseudomonas aeruginosa NCIM 5029 and Staphylococcus aureus NCIM 5021. They were specifically grown in cation-controlled Müller-Hinton broth 
(CAMHB; Himedia) at a temperature of $37^{\circ} \mathrm{C}$ with aeration for $16-$ $18 \mathrm{~h}$.

Antimicrobial compounds. The antibiotics selected for the study were amikacin, ampicillin, erythromycin, ciprofloxacin and vancomycin. Seven phenylpropanoids were selected for the study, of which four were hydroxycinnamic acids bearing hydroxyl groups ( $p$ coumaric, caffeic, chlorogenic and ferulic acids). All the antibiotics and the phenylpropanoids cinnamic, p-coumaric, caffeic and 3,4dimethoxycinnamic acids were obtained from Himedia (India). 2,4,5Trimethoxycinnamic acid was purchased from Sigma and chlorogenic and ferulic acids were from SRL. Ampicillin, amikacin and vancomycin were dissolved in water, erythromycin was dissolved in $95 \%$ ethanol and ciprofloxacin was dissolved in alkaline water. The phenylpropanoids were solubilized in DMSO. All the chemicals and solvents used in the experiments were purchased from SRL.

Microdilution method. The MIC of the antibiotics and the phenylpropanoids was determined by a microdilution method. A total of $100 \mu \mathrm{l}$ CAMHB was distributed into each well and twofold dilutions of the compounds were made (CLSI, 2006). Each microtitre well was inoculated with $10 \mu \mathrm{l}$ of the bacterial inoculum of $5 \times 10^{5}$ c.f.u. $\mathrm{ml}^{-1}$, and the plates were incubated at $37^{\circ} \mathrm{C}$ for $18 \mathrm{~h}$ under aerobic conditions. The MIC was defined as the lowest concentration of compounds that completely inhibited $(>90 \%)$ the growth of the organism as detected at $\mathrm{OD}_{600}$ and compared with that of the control. The concentration of the solvents used in the following assays was maintained at less than $3 \%$ so that no inhibition of organisms or interference occurred. The assay was repeated in quadruplicate, where the MIC is the modal value of the data.

In the chequerboard method, the antibiotic in the combination was serially diluted along the ordinate of the plate, while the phytochemical was diluted along the abscissa. The antagonistic, additive (indifferent) or synergistic effect of the phytochemical in combination with the antibiotic was determined by two methods. The first method involved plotting the data as isobolograms (Hemaiswarya \& Doble, 2009). The graph is represented with the ratio of the MIC of the phenylpropanoid in combination to the MIC of the phenylpropanoid when used alone on the $x$-axis and the ratio of the MIC of the antibiotic in combination to the MIC of the antibiotic when used alone on the $y$-axis. A straight line that connects the ratio 1.0 in the ordinate and 1.0 in the abscissa indicates the line of additivity. Location of the MIC of the combination considerably below this line (below the $95 \%$ confidence band) indicates synergy, above the line indicates antagonism and near the line indicates additive.

In the second method for identifying synergy between the phytochemical and antibiotic, the MIC of the combination is represented as the FIC (fractional inhibitory concentration) index. The FIC index $=$ FIC $\mathrm{P}+$ FIC A, where FIC $(\mathrm{P}$ or $\mathrm{A})=\mathrm{MIC}(\mathrm{P}$ or $\mathrm{A})$ in combination/MIC ( $\mathrm{P}$ or $\mathrm{A})$ alone $(\mathrm{P}=$ phenylpropanoid and $\mathrm{A}=$ antibiotic) .

If the FIC index is $\leqslant 0.5$, the interaction is synergistic, if it is $\geqslant 4.0$, it is antagonistic, and between $\geqslant 0.5$ and $\leqslant 4.0$ it is indifferent (Odds, 2003).

Time-kill curve analysis. A time-kill curve (c.f.u. as a function of time) is evaluated to study the rate and extent of reduction in bacterial burden when treated with a compound. The experiments were conducted in CAMHB for $24 \mathrm{~h}$ with 0.5 times the MIC of the phytochemical and the antibiotic used alone and in combination against all the four strains. An initial inoculum of approximately $5 \times 10^{5}$ c.f.u. $\mathrm{ml}^{-1}$ was taken for all the experiments. Samples $(0.1 \mathrm{ml})$ were collected at $0,2,4,6,8$ and $24 \mathrm{~h}$ and serially diluted in normal saline and aliquoted in duplicate onto Müller-Hinton agar plates. These plates were then incubated at $37^{\circ} \mathrm{C}$ for $24 \mathrm{~h}$. The colonies
(10-100 per plate) were counted, providing a lower limit of detection of $10^{2}$ c.f.u. $\mathrm{ml}^{-1}$ (Tam et al., 2005).

The modified McFarland method reported by Pai et al. (2008) was followed here to estimate parameters such as difference in the area under the curve (log c.f.u. as a function of time) for control and treated $(\mathrm{ABTKC})$ and maximum rate of kill $\left(K_{\max }\right)$. The rate of kill (K) was calculated as $K=\left(\log _{10} \delta T-\log _{10} \delta C\right) / \delta t$, where $\delta T$ and $\delta C$ are the differences in c.f.u. $\mathrm{ml}^{-1}$ in treatment (namely $T_{t_{1}}-T_{t_{0}}, T_{t_{2}}-T_{t_{1}}$, $T_{t_{3}}-T_{t_{2}}$, etc.) and in control (namely $C_{t_{1}}-C_{t_{0}}, C_{t_{2}}-C_{t_{1}}, C_{t_{3}}-C_{t_{2}}$, etc.), respectively, at specified time differences $\left(\delta t=t_{1}-t_{0}, t_{2}-t_{1}\right.$, $t_{3}-t_{2}$, etc.). The value of $K$ was assumed to be 0 at time zero, and $K$ for the respective $\delta t$ value was plotted (on the $y$-axis) against 2, 4, 6, 8 and $24 \mathrm{~h}$ (on the $x$-axis). The $K_{\max }$ was estimated based on visual inspection. The change in $\log _{10}$ c.f.u. $\mathrm{ml}^{-1}$ over $24 \mathrm{~h}$ was translated into the area under the time-kill curve (AUTKC) by the linear trapezoidal rule. The treatments were normalized by subtracting the treatment AUTKC from the control AUTKC to generate ABTKC. The calculated parameters between the combination and individual treatment groups were compared using ANOVA (Pai et al., 2008) and $P$-values of $<0.05$ were considered statistically significant.

Measuring bacterial membrane damage. A LIVE/DEAD BacLight kit containing SYTO-9 and propidium iodide dyes from Molecular Probes (Invitrogen) was used to assess the damage to the bacterial membrane. SYTO-9 binds to both the damaged and intact membranes, whereas propidium iodide penetrates only the damaged cell membranes. Therefore, live cells appear green and dead ones appear red. The organisms were grown overnight in CAMHB at $37{ }^{\circ} \mathrm{C}$ under aeration (200 r.p.m.). The culture was diluted 40 times with fresh CAMHB and grown to an $\mathrm{OD}_{600}$ of $0.5-0.6$. The bacterial suspension was centrifuged at $10000 \mathrm{~g}$ for $15 \mathrm{~min}$, and the cell pellet was washed once in filter-sterilized distilled water. The cell pellet was resuspended to $1 / 10$ th of its original volume and then diluted to $1: 20$ into either water or water containing the phytochemical at a concentration of $1 \mathrm{mM}$. Bacteria and compounds were incubated at room temperature $\left(\sim 25^{\circ} \mathrm{C}\right)$ on a tube rocker for $10 \mathrm{~min}$. At the end of the incubation period, a sample was removed for the determination of c.f.u., and the remaining suspension was centrifuged at $10000 \mathrm{~g}$ for $10 \mathrm{~min}$, washed once in water, and resuspended to an $\mathrm{OD}_{670}$ of 0.325 . A volume of $3 \mathrm{ml}$ of the bacterial suspension was removed and $9 \mu \mathrm{l}$ of the BacLight reagent was then added and incubated in the dark for $15 \mathrm{~min}$ at room temperature. At the end of the incubation period, green fluorescence was read at $530 \mathrm{~nm}$, and red fluorescence was read at $645 \mathrm{~nm}$ at an excitation wavelength of $485 \mathrm{~nm}$ (Jasco fluorimeter). The ratio of green to red fluorescence intensities was normalized to the untreated control and expressed as a percentage of the control (Hilliard et al., 1999)

2D-Quantitative structure-activity relationships (QSARs). The structures of the phenylpropanoids were drawn and their minimum energy conformation was determined with Cerius ${ }^{2}$ software using Universal force field (Accelrys). Two hundred and forty-nine descriptors that included topological, charge, geometrical and aromaticity indices, constitutive properties, quantum mechanics and thermodynamics were calculated for each compound. Several literature reports give a very detailed description of these descriptors (Todeschini et al., 1994; Todeschini \& Consonni, 2000; Karelson, 2000). Equations were developed between the observed membranedamaging activity of the phytochemical and the descriptors. The activity is defined as $\log [p /(100-p)]$, where $p$ is the percentage membrane damage caused by the compounds at a concentration of $1 \mathrm{mM}$. The main challenge in QSARs is to select the descriptors from this large pool that would provide the best model. The dataset was divided into training and test sets, the former consisting of five randomly chosen compounds and the latter consisting of the remainder. The model was developed using the training set and its 
predictive ability was ascertained with the data in the test set. The multilinear regression model that gave the best statistics $\left(r^{2}, r^{2}\right.$-adj., Fvalue) was selected using the genetic function technique. The predicted and experimental values were plotted and it was also checked whether all the points fell within the $95 \%$ prediction band (i.e. $\pm 2 \sigma$ standard deviation). Only one parameter model was tested, since there were only seven data points.

\section{RESULTS}

\section{Interaction of phenylpropanoids with antibiotics}

The antibacterial activities of phenylpropanoids with different classes of antibiotics were determined against Gram-positive and -negative bacteria. In all cases, the natural products had a higher MIC than the antibiotics (Supplementary Table S1 in JMM Online).

Representative isobolograms of the combination of cinnamic acid with amikacin and ampicillin against all the four micro-organisms are shown graphically in Fig. 1. A synergistic interaction between cinnamic acid and the two antibiotics (ampicillin and amikacin) was observed for all the organisms with the MIC of the combinations falling well below the line of additivity. The interaction data in the

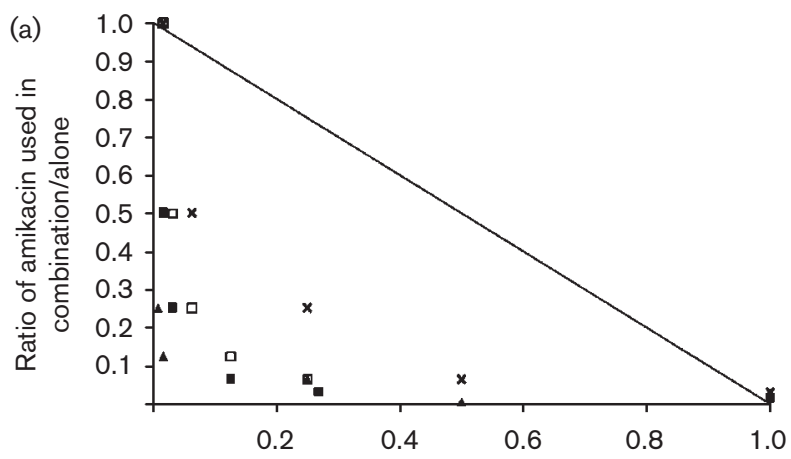

Ratio of cinnamic acid used in combination/alone

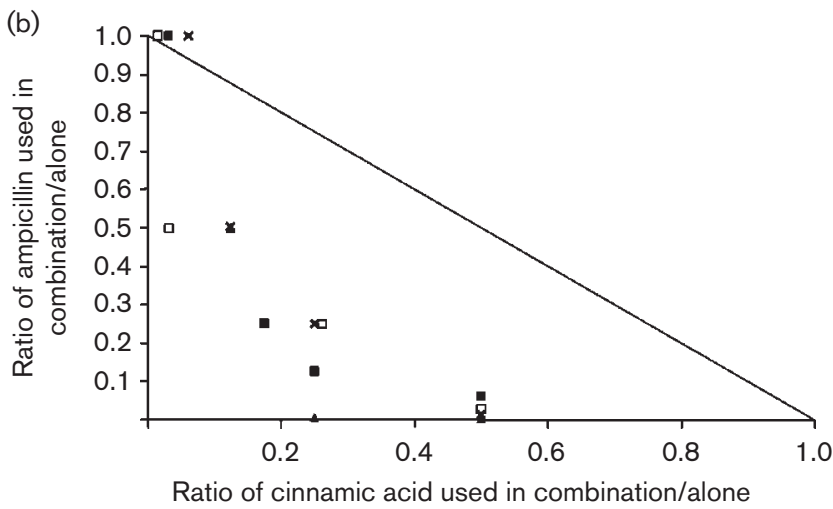

Fig. 1. Representative isobolograms depicting the interaction of cinnamic acid with (a) amikacin and (b) ampicillin against $E$. coli $(\boldsymbol{\Delta})$, Ent. aerogenes $(\square), P$. aeruginosa $(\times)$ and $S$. aureus $(\boldsymbol{\square})$. The line connecting 1.0 on the abscissa and ordinate indicates additivity. form of FIC are listed in Table 1. S. aureus was most susceptible to combinations of ferulic or cinnamic acid with antibiotics (FIC <0.5). Ferulic acid exhibited indifference in combination with ampicillin against all the micro-organisms. Cinnamic acid was synergistic with all five antibiotics against E. coli, while it was indifferent with all the antibiotics against $P$. aeruginosa. 3,4Dimethoxycinnamic acid and 2,4,5-trimethoxycinnamic acid enhanced the activity of ciprofloxacin, erythromycin and vancomycin against most of the organisms. Exceptions were noted with ampicillin against Ent. aerogenes and $P$. aeruginosa. Caffeic and chlorogenic acids did not exhibit synergy with any of the tested antibiotics.

The time-kill curves and rate-of-kill curves for E. coli treated with 0.5 times the MIC of amikacin or cinnamic acid and their combinations are shown in Figs 2 and 3, respectively. Growth of E. coli was completely inhibited when the combination was used (Fig. 2) and the corresponding $K_{\max }$ was less than the values for individual treatments (Fig. 3). A representative table (Table 2) of the time-kill parameters is given for amikacin with all the phytochemicals against E. coli. Higher ABTKC and lower $K_{\max }$ values for the combination when compared to the corresponding individual compounds indicate synergy. The ABTKC values for chlorogenic and caffeic acids when used alone were negative, indicating that these acids are enhancing growth of the micro-organisms. The parameters of all the phytochemicals in combination with antibiotics against $P$. aeruginosa were not better than those of the individual treatments. Cinnamic, $p$-coumaric and ferulic acids were synergistic with amikacin against E. coli, Ent. aerogenes and S. aureus. 3,4-Dimethoxycinnamic and 2,4,5trimethoxycinnamic acids exhibited synergy with some of the antibiotics, but there was no consistency in their interaction (data not shown).

\section{Membrane-damaging effect of phenylpropanoids}

The LIVE/DEAD BacLight assay distinguishes the intact and damaged cell membranes. The bacterial membrane damage caused by the phenylpropanoids is represented as percentage damage compared to that of control (Fig. 4). Ferulic acid was less active against Gram-negative bacteria, whereas there was nearly $50 \%$ damage in S. aureus. Highest membrane damage was noted with cinnamic acid against all the four micro-organisms. Caffeic and chlorogenic acids were able to considerably damage the membrane of all the bacteria. 3,4-Dimethoxycinnamic acid and 2,4,5-trimethoxycinnamic acid were able to damage 25 and $65 \%$ of the E. coli membrane, respectively. Trimethoxycinnamic acid showed least membrane damage against Ent. aerogenes and $P$. aeruginosa.

\section{D-QSARs}

In the present study, QSARs to predict the membranedamaging effect of the seven phenylpropanoids against all 

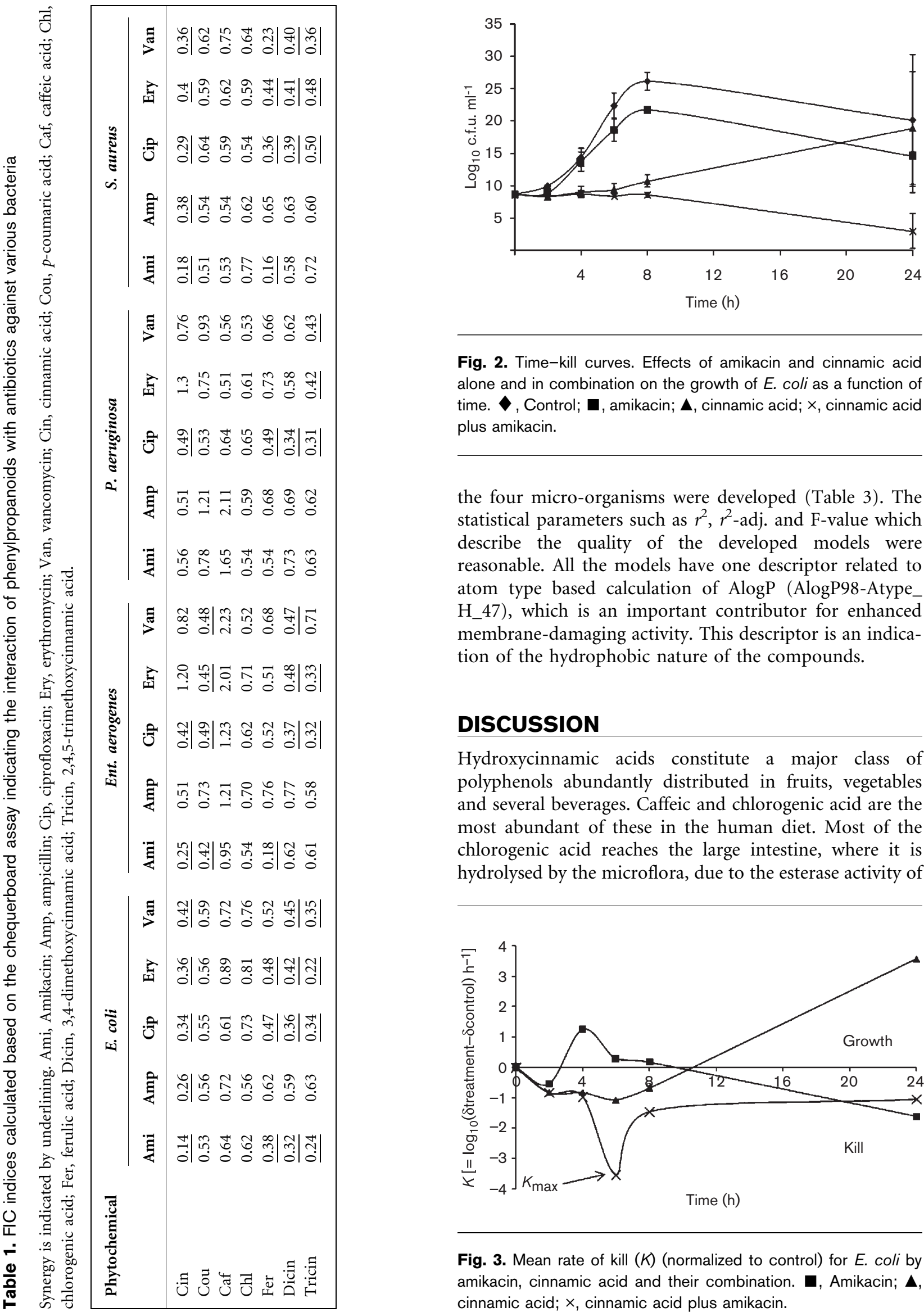

Fig. 2. Time-kill curves. Effects of amikacin and cinnamic acid alone and in combination on the growth of $E$. coli as a function of time. $\diamond$, Control; $\boldsymbol{\square}$, amikacin; $\boldsymbol{\Lambda}$, cinnamic acid; $\times$, cinnamic acid plus amikacin.

the four micro-organisms were developed (Table 3). The statistical parameters such as $r^{2}, r^{2}$-adj. and F-value which describe the quality of the developed models were reasonable. All the models have one descriptor related to atom type based calculation of AlogP (AlogP98-Atype H_47), which is an important contributor for enhanced membrane-damaging activity. This descriptor is an indication of the hydrophobic nature of the compounds.

\section{DISCUSSION}

Hydroxycinnamic acids constitute a major class of polyphenols abundantly distributed in fruits, vegetables and several beverages. Caffeic and chlorogenic acid are the most abundant of these in the human diet. Most of the chlorogenic acid reaches the large intestine, where it is hydrolysed by the microflora, due to the esterase activity of

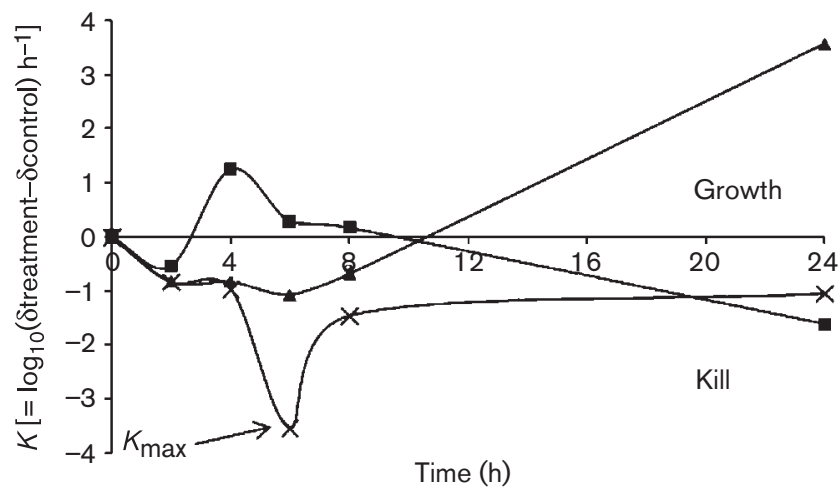

Fig. 3. Mean rate of kill $(K)$ (normalized to control) for $E$. coli by amikacin, cinnamic acid and their combination. $\mathbf{\square}$, Amikacin; $\boldsymbol{\Delta}$, cinnamic acid; $\times$, cinnamic acid plus amikacin. 
Table 2. Comparison of time-kill curve parameters for phenylpropanoid and amikacin combinations against $E$. coli

\begin{tabular}{|c|c|c|c|c|}
\hline Treatment & АВTKC & $K_{\max }$ & ABTKC & $K_{\max }$ \\
\hline Cinnamic acid & $187.9 \pm 23.4$ & $-1.1 \pm 0.3$ & $382.1 \pm 20.3^{\star}$ & $-3.5 \pm 0.9$ \\
\hline$p$-Coumaric acid & $10.54 \pm 7.92$ & $-3.9 \pm 0.5$ & $241.3 \pm 60.3^{*}$ & $4.0 \pm 0.4^{*}$ \\
\hline Caffeic acid & $-147.8 \pm 40.4$ & $-0.9 \pm 0.8$ & $-28.84 \pm 0.79 \dagger$ & $-0.1 \pm 0.3$ \\
\hline 3,4-Dimethoxycinnamic acid & $93.0 \pm 70.4$ & $-0.6 \pm 0.2$ & $173.5 \pm 86.2$ & $-1.3 \pm 1.2$ \\
\hline 2,4,5-Trimethoxycinnamic acid & $71 \pm 10.3$ & $-1.3 \pm 0.7$ & $262.0 \pm 82.1^{\star}$ & $-2.9 \pm 0.1^{\star}$ \\
\hline
\end{tabular}

${ }^{\star} P<0.05$, combination better than the individual treatments.

$\dagger P<0.05$, individual treatment better than the combination.

the latter (Plumb et al., 1999; Gonthier et al., 2003). It has been demonstrated that humans absorb about 33 and $95 \%$ of ingested chlorogenic and caffeic acids, respectively (Olthof et al., 2001). It has been shown that the unabsorbed chlorogenic acid in humans reaches the colon and is hydrolysed to caffeic and quinic acid by the microflora present there (Olthof et al., 2003). Caffeic acid is the direct product of the hydrolysis of chlorogenic acid, and ferulic and isoferulic acids are tissular metabolites formed by methylation of caffeic acid (Masri et al., 1964; Gumbinger et al., 1993). Following dehydroxylation by the colonic microflora, absorption and further metabolism in the liver and kidney, benzoic acid is formed and conjugated to glycine to form hippuric acid. About half of the ingested chlorogenic acid appears as urinary hippuric acid (Olthof et al., 2003). 3,4-Dimethoxycinnamic and 2,4,5-trimethoxycinnamic acids are unusual, and they occur as free acids in commercial green coffee beans (Clifford, 2000; Loke et al., 2009). All the phenylpropanoids and antibiotics enter the bloodstream after metabolism; therefore, there is a possibility of interaction between these compounds.

The current study indicates that there is a synergistic interaction between various phenylpropanoids and antibiotics against Gram-positive and -negative bacteria. The antibiotics in the study were chosen based on their different mechanisms of action and their association with some side effects or toxicity. For example, aminoglycosides

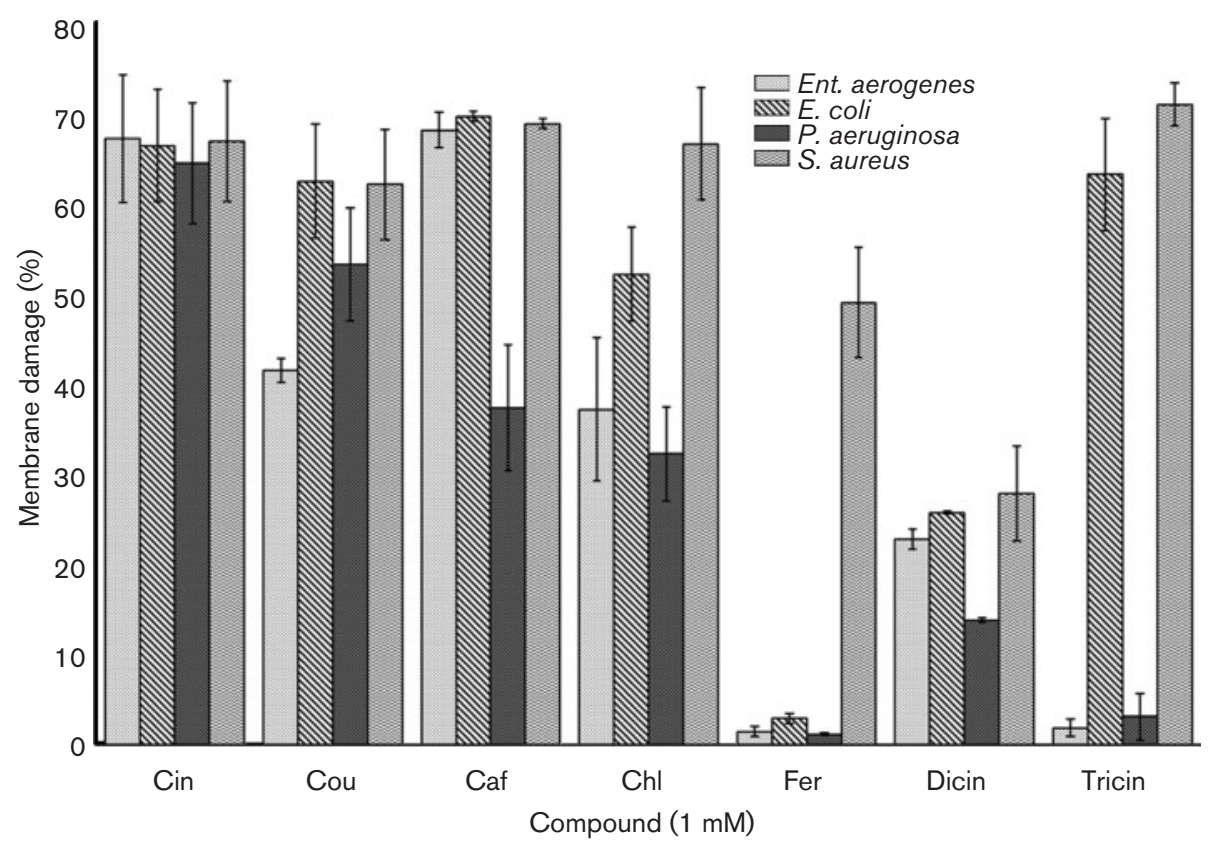

Fig. 4. Effect of $1 \mathrm{mM}$ phenylpropanoids on bacterial membrane damage based on the LIVE/DEAD BacLight kit. 
Table 3. 2D-OSAR models describing membrane-damaging activity of phenylpropanoids against micro-organisms

The activity is defined as $\log [p /(100-p)]$ where $p$ is percentage value.

\begin{tabular}{|lcccc|}
\hline $\begin{array}{l}\text { Micro- } \\
\text { organism }\end{array}$ & Model & $r^{2}$ & $r^{2}$-adj. & F-value \\
\hline E. coli & $0.39-0.09$ (Atype_H_47) & 0.86 & 0.81 & $17.79^{*}$ \\
Ent. aerogenes & $0.25-0.09$ (Atype_H_47) & 0.7 & 0.59 & $6.67 \dagger$ \\
P. aeruginosa & $0.18-0.09$ (Atype_H_47) & 0.81 & 0.74 & $12.42^{*}$ \\
S. aureus & $0.39-0.01$ (Atype_H_47) & 0.63 & 0.51 & $5.09 \dagger$ \\
\hline
\end{tabular}

${ }^{\star} P \leqslant 0.01$.

$\dagger P \leqslant 0.05$.

(amikacin) have undesirable side effects such as ototoxicity and/or nephrotoxicity in vivo (Paglia et al., 1989). The interaction with phenylpropanoids generally enhances the activity of the antibiotics. Based on the FIC indices, it could be said that chlorogenic and caffeic acids were indifferent in nature with all the antibiotics against both Grampositive and -negative bacteria. The major metabolite ferulic acid was synergistic with amikacin and ciprofloxacin against most of the micro-organisms. Ampicillin and the four hydroxycinnamic acid combinations showed indifference and combinations of hydroxycinnamic acids with amikacin or ciprofloxacin exhibited synergy. The methoxy forms of cinnamic acid including ferulic, 3,4-dimethoxycinnamic and 2,4,5-trimethoxycinnamic acid were synergistic with some of the antibiotics. Rastogi et al. (1994, 1998) observed that trans-cinnamic acid enhanced the activity of isoniazid, rifampicin, ofloxacin, amikacin and clofazimine against Mycobacterium avium and Mycobacterium tuberculosis.

Complex methods of interaction have been adopted by researchers to determine the synergy of antibacterial combinations. A change of greater than $2 \log _{10}$ c.f.u. $\mathrm{ml}^{-1}$ in the bacterial viable count at $24 \mathrm{~h}$ in the presence of a combination of the drugs relative to the count with the most effective single drug is defined as synergy (Saiman, 2007). This definition restricts the evaluation to a single point. Alternatively, the bacterial burden at different time points can be calculated, which leads to an increased probability of finding large differences. The area under the curve concept provides us additional details such as the killing rate and phase of regrowth. In the chequerboard method, we found that the methoxy forms of cinnamic acid (namely ferulic, 3,4-dimethoxy and 2,4,5-trimethoxycinnamic acids) exhibited synergy in combination with ciprofloxacin against $P$. aeruginosa. None of the other four phytochemicals exhibited synergy. However, in time-kill curve studies, none of the combinations indicated synergy against $P$. aeruginosa. Chequerboard assay and time-kill curve studies indicated that chlorogenic and caffeic acids do not act in synergy with any of the antibiotics against all the organisms. This could possibly be explained by the fact that both these hydroxycinnamic acids are metabolized by the bacteria. The ABTKC for the chlorogenic and caffeic acids was negative, probably because these phenylpropanoids are used by many micro-organisms as carbon sources for their growth (Couteau et al., 2001; Aresta et al., 2010). Based on the time-kill curve studies, it could be concluded that cinnamic, $p$-coumaric and ferulic acids were the most active in combination with the antibiotics against most of the organisms. S. aureus was the most sensitive organism to the combinations.

All the acids had moderate to high membrane-damaging activity. Membranes of Ent. aerogenes and $P$. aeruginosa were slightly resistant to damage because of their increased surface hydrophobicity. Hydrophobicity of the bacterial surface seems to play a major role in the damage caused by the compounds. The hydrophobicity of the Gram-negative organisms in this study can be ordered as E. coli<Ent. aerogenes $<P$. aeruginosa (Hemaiswarya \& Doble, 2009). We observed that the order of damage in the Gramnegative bacteria was $E$. coli $>$ Ent. aerogenes $>P$. aeruginosa, correlating exactly with their surface hydrophobicity. Cinnamic, chlorogenic and caffeic acid were able to damage the bacterial membrane more than the other compounds. Caffeic and $p$-coumaric acid were reported by other researchers to cause 44 and $59 \%$ membrane damage, respectively, in a Gram-positive bacterium, Oenococcus oeni (Campos et al., 2009). The antifungal mode of action of chlorogenic acid against Candida albicans was analysed by flow cytometry using $\mathrm{DiBAC}_{4}(3)$ and simultaneously changes in its membrane dynamics were monitored using 1,6-diphenyl-1,3,5-hexatriene. The study suggested that chlorogenic acid may exert antifungal activity by disrupting the structure of the cell membrane (Sung \& Lee, 2010). In our studies, we observed that although they both caused membrane damage, there was no synergistic interaction with any of the antibiotics. This could be explained by the fact that the LIVE/DEAD BacLight experiments were done by preincubating cells for $10 \mathrm{~min}$ with the compound, whereas the synergy studies were performed at the 18th hour of incubation. An in vitro fermentation model with human faecal microbiota to study the microbial metabolism of caffeic acid and its esters, namely chlorogenic acid and caftaric acid, has shown that they disappeared within $2 \mathrm{~h}$ (Gonthier et al., 2006). Since chlorogenic acid is rapidly metabolized, no positive interaction was noted with the antibiotics. The high membrane damage noted in the LIVE/DEAD BacLight experiments in our study can be attributed to the fact that resting cells were taken for the assay. It has also been demonstrated that some bacterial strains may respond to the presence of phenolic acids by increasing the unsaturated fatty acid content (and presumably the fluidity) of the cell membrane (Rozès \& Peres, 1998). Under resting-cell conditions, bacteria might not be able to counteract the combined effect of phenolic acids and ethanol on the cytoplasmic membrane (Campos et al., 2009). A non-metabolizable form of chlorogenic acid could probably act as a better membrane-permeabilizing agent. 
Synergy is believed to be brought about by a combination of drugs because of their action on one or more different targets in the metabolic pathway. The current study shows that interaction of phenylpropanoids is primarily with the membrane of the bacterial cell. Due to this action, they are able to enhance the activity of the antibiotics amikacin, ampicillin, erythromycin, ciprofloxacin and vancomycin. The main target of ampicillin and vancomycin is to inhibit cell wall synthesis. The other antibiotics (ciprofloxacin, erythromycin, amikacin), in addition to their specific target site action, also damage the cell membrane (Campos et al., 2006). Only with exact knowledge of the mechanism underlying the synergistic effects will it be possible to develop a new generation of safe and standardized drug combinations with higher efficacy than the current ones (Wagner \& Ulrich-Merzenich, 2009).

We observed (Table 3) that all the models describing the membrane-damaging activity have Atype_H_47 as the descriptor. This descriptor denotes the hydrogen attached to the $\mathrm{C}_{\mathrm{sp3}}^{1}$ and $\mathrm{C}_{\mathrm{sp2}}^{0}$ atoms, where the subscript represents hybridization and the superscript its formal oxidation number. The atom classification is an attempt to discretize the electronic effects, solvent accessibility and so forth of an atom from a topological consideration. These properties are the most critical in determining the relative contribution of different atom types to $\log P$ for small organic molecules with relatively few degrees of freedom. Thus Atype_H_47 is the most atomic parameter which contributes to $\log P$, which is a well-known measure of molecular hydrophobicity (also known as lipophilicity). It is used to assess biological properties relevant to drug action, such as lipid solubility, tissue distribution, receptor binding, cellular uptake, metabolism and bioavailability. The AlogP98 descriptor is an implementation of the atomtype-based $\mathrm{A} \log \mathrm{P}$ method using the latest published set of parameters (Ghose et al., 1998). Each AlogP98 atom-type value represents the number of atoms of that type in the molecule and Cerius $^{2}$ calculates 120 atom types. The negative slope of Atype_H_47 in this equation shows that its presence is not essential for the activity.

The phenylpropanoids have been shown to possess antibacterial activity and enhance the activity of a heterogeneous group of antibiotics in a non-specific manner. The importance of these acids in damaging the bacterial cell membrane is shown by the LIVE/DEAD BacLight assay. The models developed here possess promising predictive ability to elucidate the relationship between the structure of the compounds and membranedamaging activity so that they can be used to design more potent phenylpropanoid derivatives. These findings can lead to new treatment strategies and could also pave the way to the reduction of the amount of antibiotics required, particularly against Gram-negative bacteria. A reduction in the antibiotic amount can eventually lead to reduction in their toxicity and side effects caused to the patients. The next focus should be on the relative pharmacokinetic and pharmacodynamic behaviour of these natural compounds with reference to these antibiotics and when used in combination.

\section{REFERENCES}

Aresta, M., Acquaviva, M. I., Baruzzi, F., Lo Noce, R. M., Matarante, A., Narracci, M., Stabili, L. \& Cavallo, R. A. (2010). Isolation and characterization of polyphenols-degrading bacteria from olive-mill wastewaters polluted soil. World J Microbiol Biotechnol 26, 639647.

Betrosian, A. P. \& Douzinas, E. E. (2009). Ampicillin-sulbactam: an update on the use of parenteral and oral forms in bacterial infections. Expert Opin Drug Metab Toxicol 5, 1099-1112.

Campos, M. A., Morey, P. \& Bengoechea, J. A. (2006). Quinolones sensitize Gram-negative bacteria to antimicrobial peptides. Antimicrob Agents Chemother 50, 2361-2367.

Campos, F. M., Couto, J. A., Figueiredo, A. R., Tóth, I. V., Rangel, A. O. S. S. \& Hogg, T. A. (2009). Cell membrane damage induced by phenolic acids on wine lactic acid bacteria. Int J Food Microbiol 135, 144-151.

Clifford, M. N. (1999). Chlorogenic acids and other cinnamates nature, occurrence and dietary burden. J Sci Food Agric 79, 362-372.

Clifford, M. N. (2000). Chlorogenic acids and other cinnamates nature, occurrence, dietary burden, absorption and metabolism. J Sci Food Agric 80, 1033-1043.

CLSI (2006). Methods for Dilution Antimicrobial Susceptibility Tests for Bacteria that Grow Aerobically, 7th edn. Approved Standard M7-A7. Wayne, PA: Clinical and Laboratory Standards Institute.

Couteau, D., McCartney, A. L., Gibson, G. R., Williamson, G. \& Faulds, C. B. (2001). Isolation and characterization of human colonic bacteria able to hydrolyse chlorogenic acid. J Appl Microbiol 90, 873881.

Ghose, A. K., Viswanadhan, V. N. \& Wendoloski, J. J. (1998). Prediction of hydrophobic (lipophilic) properties of small organic molecules using fragmental methods: an analysis of ALOGP and CLOGP methods. J Phys Chem 102, 3762-3772.

Gonthier, M.-P., Verny, M.-A., Besson, C., Rémésy, C. \& Scalbert, A. (2003). Chlorogenic acid bioavailability largely depends on its metabolism by the gut microflora in rats. J Nutr 133, 1853-1859.

Gonthier, M.-P., Rémésy, C., Scalbert, A., Cheynier, V., Souquet, J.-M., Poutanen, K. \& Aura, A.-M. (2006). Microbial metabolism of caffeic acid and its esters chlorogenic and caftaric acids by human faecal microbiota in vitro. Biomed Pharmacother 60, 536-540.

Gumbinger, H. G., Vahlensieck, U. \& Winterhoff, H. (1993). Metabolism of caffeic acid in the isolated perfused rat liver. Planta Med 59, 491-493.

Hemaiswarya, S. \& Doble, M. (2009). Synergistic interaction of eugenol with antibiotics against Gram negative bacteria. Phytomedicine 16, 997-1005.

Hilliard, J. J., Goldschmidt, R. M., Licata, L., Baum, E. Z. \& Bush, K. (1999). Multiple mechanisms of action for inhibitors of histidine protein kinases from bacterial two-component systems. Antimicrob Agents Chemother 43, 1693-1699.

Karelson, M. (2000). Molecular Descriptors in QSAR/QSPR. New York: Wiley Interscience.

Korkina, L. G. (2007). Phenylpropanoids as naturally occurring antioxidants: from plant defense to human health. Cell Mol Biol (Noisy-le-grand) 53, 15-25.

Loke, W. M., Jenner, A. M., Proudfoot, J. M., McKinley, A. J., Hodgson, J. M., Halliwell, B. \& Croft, K. D. (2009). A metabolite profiling 
approach to identify biomarkers of flavonoid intake in humans. J Nutr 139, 2309-2314.

Masri, M. S., Robbins, D. J., Emerson, O. H. \& DeEds, F. (1964). Selective para- or meta-O-methylation with catechol $O$-methyl transferase from rat liver. Nature 202, 878-879.

Odds, F. C. (2003). Synergy, antagonism, and what the chequerboard puts between them. J Antimicrob Chemother 52, 1.

Olthof, M. R., Hollman, P. C. H. \& Katan, M. B. (2001). Chlorogenic acid and caffeic acid are absorbed in humans. J Nutr 131, 66-71.

Olthof, M. R., Hollman, P. C., Buijsman, M. N., van Amelsvoort, J. M. \& Katan, M. B. (2003). Chlorogenic acid, quercetin-3-rutinoside and black tea phenols are extensively metabolized in humans. J Nutr 133, 1806-1814.

Paglia, P., Molinari, G., Pesce, A. \& Debbia, E. A. (1989). Dactimicin, a new aminoglycoside: in vitro activity, post-antibiotic effect and interaction with other antibiotics. Eur J Clin Microbiol Infect Dis 8, 639-643.

Pai, M. P., Samples, M. L., Mercier, R. C. \& Spilde, M. N. (2008). Activity and ultrastructural effects of antifungal combinations against simulated Candida endocardial vegetations. Antimicrob Agents Chemother 52, 2367-2376.

Plumb, G. W., Garcia-Conesa, M. T., Kroon, P. A., Rhodes, M., Ridley, S. \& Williamson, G. (1999). Metabolism of chlorogenic acid by human plasma, liver, intestine and gut microflora. J Sci Food Agric 79, 390-392.

Rastogi, N., Goh, K. S., Wright, E. L. \& Barrow, W. W. (1994). Potential drug targets for Mycobacterium avium defined by radiometric druginhibitor combination techniques. Antimicrob Agents Chemother 38, 2287-2295.
Rastogi, N., Goh, K. S., Horgen, L. \& Barrow, W. W. (1998). Synergistic activities of antituberculous drugs with cerulenin and trans-cinnamic acid against Mycobacterium tuberculosis. FEMS Immunol Med Microbiol 21, 149-157.

Rozès, N. \& Peres, C. (1998). Effects of phenolic compounds on the growth and the fatty acid composition of Lactobacillus plantarum. Appl Microbiol Biotechnol 49, 108-111.

Saiman, L. (2007). Clinical utility of synergy testing for multidrugresistant Pseudomonas aeruginosa isolated from patients with cystic fibrosis: 'the motion for'. Paediatr Respir Rev 8, 249-255.

Sung, W. S. \& Lee, D. G. (2010). Antifungal action of chlorogenic acid against pathogenic fungi, mediated by membrane disruption. Pure Appl Chem 82, 219-226.

Tam, V. H., Schilling, A. N. \& Nikolaou, M. (2005). Modelling time-kill studies to discern the pharmacodynamics of meropenem. J Antimicrob Chemother 55, 699-706.

Todeschini, R. \& Consonni, V. (2000). Handbook of Molecular Descriptors. Weinheim: Wiley-VCH.

Todeschini, R., Lasagni, M. \& Marengo, E. (1994). New molecular descriptors for 2D and 3D structures. Theory. J Chemom 8, 263272.

Tonari, K., Mitsui, K. \& Yonemoto, K. (2002). Structure and antibacterial activity of cinnamic acid related compounds. J Oleo Sci 51, 271-273.

Wagner, H. \& Ulrich-Merzenich, G. (2009). Synergy research: approaching a new generation of phytopharmaceuticals. Phytomedicine 16, 97-110. 